\title{
Bourdieu en la educación científica: consecuencias para la enseñanza y la investigación.
}

\author{
Lima Junior, Paulo \\ Pinheiro, Nathan \\ Ostermann, Fernanda
}

Universidade Federal do Rio Grande do Sul

Resumen: A lo largo de las últimas décadas, el desarrollo y la consolidación de un abordaje sociocultural a la educación científica han dado lugar a un creciente interés por referencias de ámbitos como la sociología de la ciencia y la sociología de la educación del sociólogo francés Pierre Bourdieu (1930 - 2002). Con el propósito general de contribuir al avance de la apropiación de la obra de Bourdieu por parte de la investigación en educación científica, el presente artículo presenta algunas de las ideas más importantes de este autor y discute sus consecuencias para la enseñanza de ciencias y su investigación.

Palabras clave: Pierre Bourdieu, Sociología de la Ciencia, Sociología de la Educación, Educación Científica.

Abstract: Over the last decades, the development and establishment of a sociocultural approach to science education have led to an increasing interest in other theoretical frameworks such as the sociology of science and the sociology of education of Pierre Bourdieu (1930-2002). With the purpose of contributing to the advancement of the appropriation of Bourdieu's work by research in science education, this paper presents some of the most important ideas of this author and discusses their implications for science education and research.

Key Words: Pierre Bourdieu, Sociology of Science, Sociology of Education, Science Education.

(Fecha de recepción: marzo, 2012, y de aceptación: septiembre, 2012)

DOI: 10.7203/DCES.26.1925 


\section{Introducción}

A lo largo de las últimas décadas, el desarrollo y la consolidación de un abordaje sociocultural a la educación científica (Carter, 2008; Lemke, 2001) han dado lugar a un creciente interés por referencias de ámbitos como la sociología de la ciencia y la sociología de la educación. Entre esas referencias se destaca el conjunto de las contribuciones del sociólogo francés Pierre Bourdieu (1930 - 2002), una de las figuras más eminentes de la sociología moderna y, posiblemente, el científico social del siglo XX más citado del mundo.

A partir de la década del 60 , Bourdieu tuvo el mérito de formular una explicación bien fundamentada teórica y empíricamente de las relaciones entre desigualdades de clase y desigualdades escolares. Hasta mediados del siglo $\mathrm{XX}$ predominaba un espíritu optimista según el cual la universalización del acceso a la educación conduciría a la superación de la sociedad aristocrática, basada en el nacimiento, y al establecimiento de una sociedad meritocrática, en la cual las diferencias sociales serían debidas al mérito individual. Se suponía que la universalización de la educación pública otorgaría oportunidades efectivas de alcanzar mejores condiciones de vida a los individuos nacidos en el seno de familias de clase popular. Desde esta perspectiva, la escuela sería una institución neutra que seleccionaría sus alumnos en base a criterios raciona- les, contribuyendo así al aumento de la movilidad social.

A fines de la década del 50 se publicó una serie de grandes estudios cuantitativos financiados por los gobiernos inglés, norteamericano y francés que entraban en contradicción con aquellas expectativas. En dichos estudios se mostraba que el éxito escolar no estaría ligado únicamente a las aptitudes individuales sino también al origen social del estudiante ${ }^{1}$ (Nogueira \& Nogueira, 2009). Posteriormente, el fracaso del proyecto de establecimiento de la meritocracia por medio de la expansión del sistema escolar llegó a ojos del gran público: si bien amplios sectores de la sociedad tradicionalmente excluidos de la escuela concluían hora masivamente los estudios secundarios -lo cual alimentaba sus esperanzas de ascenso social-, se encontraban con un mercado de trabajo que ya no tenía lugares vacantes para el grado de formación que ahora poseían. En síntesis, los resultados de investigación mencionados y la gran insatisfacción popular con el sistema educativo -que contribuyó a la formación del importante movimiento de contestación que estalló en Francia en 1968- constituyen el contexto original de la producción académica de Bourdieu.

La obra de Bourdieu está marcada por un estilo que se revela antidogmático en diversos aspectos (Nogueira \& Nogueira, 2009), ya que rechazó: (1) tanto el empirismo positivista

1 También en la literatura contemporánea hay evidencias varias de la relación entre origen social y éxito escolar en ciencias, entre las que destaca el informe ejecutivo del Programme for International Student Assessment (PISA) referido al año 2006 (OECD, 2007). 
de la sociología norteamericana como la alternativa marxista; (2) todos los monismos metodológicos, valiéndose de la más diversas herramientas de recolección de datos; (3) tanto el subjetivismo fenomenológico, que no toma en consideración el condicionamiento social de los individuos, como el objetivismo de aquellos abordajes en que las acciones de los sujetos aparecen como completamente determinadas por la estructura social.

Bourdieu se caracteriza además por haber emprendido estudios en diversas esferas de las ciencias sociales, abordando temas tan distintos como religión, arte, escuela, literatura, prensa y ciencia. Con sus investigaciones, revela que es posible abordar temas radicalmente diferentes utilizando herramientas teóricas y metodológicas muy similares, sin por ello dejar escapar las particularidades de cada uno de los temas. Así, al mismo tiempo que ofrece una teoría sobre la organización de la sociedad en clases y sobre las relaciones entre las desigualdades de clase y las desigualdades escolares, Bourdieu también provee elementos para comprender el funcionamiento de los diversos campos de la actividad humana y, en particular, la ciencia. De hecho, este carácter integrador de la obra de Bourdieu ha con- tribuido decisivamente a que una cantidad cada vez mayor de investigadores, guiados por los más diversos objetivos, se movilice en torno de la apropiación y actualización del cuadro teórico bourdiano.

Con el propósito general de contribuir al avance de la apropiación de la obra de Bourdieu por parte de la investigación en educación científica, el presente artículo presenta algunas de las ideas más importantes de este autor y discute sus consecuencias para la enseñanza de ciencias y su investigación.

\section{La violencia simbólica y su disimu- lación}

El punto de partida de la teoría de Bourdieu sobre el sistema educativo (Bourdieu \& Passeron, 2009) es que toda acción pedagógica, es decir, cualquier acción que pretende enseñar algo a alguien, es objetivamente una violencia simbólica ${ }^{2}$, y ello en un doble sentido: (1) porque aquello que la acción pedagógica enseña (una cultura o cierta característica cultural) es siempre arbitrario; (2) porque, dado que la cultura es arbitraria, la legitimidad de la acción pedagógica depende siempre en cierta

2 Se define la violencia simbólica como el poder de imposición de significaciones y de su legitimidad (Bourdieu \& Passeron, 2009). En efecto, para quien se acerca por primera vez a su obra, algunos conceptos de Bourdieu pueden parecer impregnados de connotación moral, en el sentido en que, por ejemplo, la -práctica disimulada de violencia-difícilmente podría ser atribuida a una persona de buena índole. No obstante, Bourdieu advierte que la peor lectura que se puede hacer de su texto es una lectura moralizante que atribuya la violencia simbólica de la acción pedagógica a la acción intencional de los individuos, cuando en realidad lo que se intenta decir es, justamente, que toda acción pedagógica es inevitablemente una violencia simbólica (independientemente de que haya o no -buena fe- por parte de los sujetos). 
medida de un poder arbitrario y de la disimulación de ese carácter arbitrario.

La primera confusión que es necesario evitar respecto de esta concepción acerca del carácter arbitrario de la cultura y el poder tiene que ver con que, en Bourdieu, arbitrario no quiere decir aleatorio. De hecho, ninguna cultura real podría resultar de una combinación rigurosamente aleatoria de significaciones, pues toda cultura necesita ser inteligible como condición de su perpetuación. El argumento de Bourdieu es que la cultura inculcada en una acción pedagógica tiene siempre un carácter arbitrario en la media en que siempre involucra elecciones que nunca se justifican completamente a partir de principios (lógicos, filosóficos, naturales) de carácter universal (2009, pp. 29-30). Si comparamos diferentes sistemas de relaciones de fuerza (por ejemplo, el estado teocrático islámico, la organización tradicional en castas en la India y las democracias capitalistas occidentales) encontramos que no existe un principio universal que justifique las relaciones de fuerza que se establecen dentro de esos sistemas. De manera semejante, si comparamos sistemas simbólicos concurrentes (por ejemplo, dos religiones diferentes) podemos captar con mayor claridad lo que hay de arbitrario en cada uno de ellos.

En cuanto a las acciones pedagógicas constitutivas de la educación científica, las elecciones que dan su carácter arbitrario a la cultura científica escolar tienen lugar tanto en el ámbito del delineamiento curricular (que implica siempre una selección parcialmente injustificada de ciertas ideas científi- cas en detrimento de otras) como de la propia construcción de conocimiento científico (que nunca se justifica completamente por los argumentos teóricos o la evidencia empírica alegada, ya que también entran en juego las relaciones de fuerza que se construyen dentro del campo científico). Así, el carácter arbitrario de la educación científica puede verse tanto en el contraste entre directrices curriculares distintas (explícitamente escritas en documentos oficiales o implícitamente inscritas en los libros didácticos) como por el análisis comparativo de teorías científicas concurrentes. A causa de esta inevitable arbitrariedad en toda cultura (ya sea que resulte de una selección orientada a la enseñanza o que, simplemente y de modo más general, constituya los rasgos característicos de un determinado sujeto o grupo de sujetos) nos referimos a las diferentes culturas como arbitrarios culturales.

En cuanto a la enseñanza de Física en particular, es importante destacar que una de sus características históricas ha sido la de restringir el contacto de los alumnos con una única teoría hegemónica en cada dominio disciplinar, y este modo (arbitrario) de construcción curricular está íntimamente relacionado con la disimulación del carácter arbitrario de la propia Física. De hecho, si bien existen sistemas teóricos alternativos al de Newton para la mecánica o al de Maxwell-Lorentz para el electromagnetismo (Assis, 1995; 1998), estas teorías han conservado la hegemonía en sus respectivos dominios a lo largo de la historia. La concepción de Bourdieu acerca de la violencia simbólica y de 
la necesidad de disimulación del doble carácter arbitrario de la acción pedagógica ayuda a comprender críticamente esa situación y permite extraer consecuencias para la construcción de nuevos currículos en educación científica.

\section{Sobre el funcionamiento de los cam- pos}

No es difícil constatar que algunos arbitrarios culturales alcanzan más prestigio que otros. Véanse por ejemplo las diferencias en el tratamiento dispensado en la escuela al arte erudito $\mathrm{y}$ al arte de masas; a las religiones y a las sectas; a la lengua culta y a la coloquial; a la ciencia y a la creencia. $\mathrm{Si}$ bien la desigualdad de esas relaciones no es consensual, tales distinciones son comunes y realizan objetivamente la valorización de ciertos arbitrarios culturales en detrimento de otros.

La clasificación y jerarquización de los bienes simbólicos que constituyen los diferentes arbitrarios culturales es siempre objeto de disputa entre diversos sujetos y grupos. Esta disputa se da en diferentes esferas de la vida social, que Bourdieu denomina campos. Se llama campo a cualquier esfera de la actividad humana específicamente encargada de producir, clasificar, jerarquizar y distribuir una clase determinada de bienes (Bourdieu, 2003). Son ejemplos: el campo artístico (Bourdieu, 2009a), el religioso (Bourdieu, 2009b), el burocrático (Bourdieu, 1996a) y el científico (Bourdieu, 2004).

Al mismo tiempo que existen leyes generales aplicables a todos los campos (Bourdieu, 2003), cada campo posee una lógica propia de funcionamiento que lo distingue de los otros. Como consecuencia de esta autonomía relativa ${ }^{3}$ de los campos, lo que alguien puede acumular en un campo y que le garantiza privilegios en el mismo (prestigio, bienes y habilidades específicas del campo), no siempre se traduce de modo directo e inmediato en privilegios semejantes en otro campo. Es decir, un periodista puede ser hábil e influyente pero presentar una participación política periférica y poco calificada. Al mismo tiempo, es posible que un compositor que goza de prestigio en el campo musical tenga considerables ventajas al decidir publicar una novela en virtud del prestigio y la habilidad que acumuló como compositor, aun si no está inmediatamente calificado para actuar en el campo literario.

Según Bourdieu, el grado de autonomía de los campos no permanece siempre igual. Por ejemplo, los cambios socia-

\footnotetext{
${ }^{3}$ La concepción de Bourdieu sobre la autonomía relativa de los campos cumple una función doblemente negativa en su teoría (Bourdieu, 2004). En primer lugar, rechaza la tradición de investigación, generalmente atribuida a los marxistas, en que los fenómenos de un campo (por ejemplo, los avances de la termodinámica en el periodo de la revolución industrial) son reducidos a procesos que tienen lugar en el campo económico (Hobsbawn, 2009). Al mismo tiempo, Bourdieu se encarga de criticar las tradiciones que consideran cada campo como totalmente libre de interferencias externas, tal como hace Lakatos (1989) con el campo científico al distinguir entre historia interna y externa, y explicar el desarrollo científico preferencialmente a partir de la contrastación empírica de las teorías.
} 
les ocurridos a partir del renacimiento contribuyeron a que el campo artístico esté cada vez menos sujeto a las demandas éticas y estéticas de la aristocracia y la Iglesia (Bourdieu, 2009a). De hecho, ese proceso de autonomización marca la consolidación del campo como tal y es sumamente importante para la comprensión de su génesis histórica. Mientras que la casi totalidad de la producción intelectual en ciencias sociales es eventualmente calificada o descalificada como de izquierda o de derecha, el criterio no se aplica con la misma facilidad en Física o Química, lo cual muestra que campos distintos pueden presentar grados diferentes de autonomía en un mismo momento histórico. De hecho, en la actualidad es común la descalificación del creacionismo en Biología con el argumento de que se trata de una explicación proveniente del campo religioso y no del científico (aunque no siempre los campos de la ciencia de la naturaleza hayan gozado del grado de autonomía actual) (Bourdieu, 2004).

También es importante destacar que, al clasificar los bienes producidos por los agentes de un campo, los propios agentes se clasifican unos a otros indirectamente. Por ejemplo, al mismo tiempo que se eligen los periódicos influyentes, los científicos que acumulan publicaciones en esos periódicos tienden a acumular prestigio y se hacen más influyentes en sus respectivos campos. De este modo, reconocer que las relaciones de fuerza que se establecen en el interior de un campo están relacionadas con la acumulación de bienes producidos y valorizados en el mismo (por ejemplo, contribuciones científicas influyentes, condecoraciones militares, cargos y mandatos en la gestión pública) conduce a la definición de formas de capital específicas de cada campo. Así, podemos decir que un cantante posee un elevado capital musical si, en virtud de la acumulación de bienes producidos y valorizados en el campo musical (tales como composiciones premiadas en festivales, demostraciones de reconocimiento de parte de sus pares o del gran público, colaboraciones con otros músicos reconocidos, relaciones favorables con productores y grabadoras), goza de prestigio e influencia en dicho campo.

\section{Formas de capital y clase social}

A lo largo de la historia, aunque de manera siempre recontextualizada, el sistema escolar ha clasificado sus arbitrarios culturales a partir de jerarquías que se producen en muchos otros campos (académico, literario, artístico, burocrático, religioso). Así, tanto para el análisis del sistema escolar como para los análisis que no se restringen a un único campo, es importante definir una forma general de capital cultural que, constituida por los capitales específicos de cada uno de esos campos, garantiza a sus portadores unos privilegios mínimos en diversos contextos sociales. De este modo, posee más capital cultural el individuo que se caracteriza por rasgos culturales de alto valor en uno o más campos asociados a la producción cultural y que le confieren reconocimiento y respeto (tales como dominio del patrón culto de la lengua, erudición, buen gusto estético, conocimiento científico) ${ }^{4}$. 
Por lo tanto, encontramos que el capital cultural es, al mismo tiempo, producto de disputas entre los grupos por la clasificación en el dominio simbólico y criterio de clasificación de los individuos según su nivel cultural ${ }^{5}$. Según Bourdieu (1997), habría además otras dos formas fundamentales de capital, que sirven también como criterio de clasificación de los individuos y que, junto con el capital cultural, definen la posición social del sujeto en las relaciones de dominación ${ }^{6}$. El primero es el conocido capital económico, que refiere a títulos de propiedad, bienes materiales y todo lo que pueda convertirse directa e inmediatamente en dinero. El segundo es el capital social, que corresponde al conjunto de relaciones personales del sujeto y a la influencia de esas relaciones.

Afirmar que la posición de los sujetos se ve definida por estos tres tipos de capital (cultural, económico y social) significa que los mismos definen rela- ciones de fuerza distintas y que, por lo tanto, los tres deben ser tenidos en cuenta en la definición de las clases sociales ${ }^{7}$. Así, aun si un sujeto posee un capital económico mediano, puede ser considerado miembro de la élite si posee un capital cultural suficientemente elevado. Del mismo modo, un sujeto puede lograr acumular un capital económico considerable sin ser aceptado por las élites a causa de la escasez de su capital cultural. En síntesis, la estructura de las relaciones de clases en Bourdieu -diferente de la tradición economicista, que clasifica a los individuos por su renta- es necesariamente multidimensional (Bourdieu, 1996b).

Al igual que los campos, las tres formas de capital son relativamente autónomas, ya que cada una puede ser invertida (de manera no inmediata) en la adquisición de las otras. Por otra parte, en eso consiste uno de los mayores potenciales de generalización del con-

${ }^{4}$ Es importante agregar que el capital cultural existe en tres estados (Bourdieu, 2008b): (1) incorporado, que remite a todo lo inmaterial que forma parte de la formación cultural del sujeto (sus gustos, creencias, habilidades y disposiciones prácticas de alto valor general); (2) objetivado, que se refiere a los bienes culturales con existencia material (tales como libros, cuadros, esculturas) y suponen siempre, para su apropiación efectiva, algún capital incorporado; y (3) institucionalizado, que se refiere a los diplomas y certificados escolares, que poseen un valor de mercado más definido que los elementos de los otros estados y denotan un reconocimiento institucional del capital cultural incorporado por el individuo.

${ }^{5}$ Debe quedar claro que esa clasificación no debe ser naturalizada. Por el argumento precedente, la definición de un mayor o menor capital cultural se basa en criterios esencialmente arbitrarios.

${ }^{6} \mathrm{~A}$ las tres formas generales de capital podríamos agregar el llamado capital simbólico, que denota el prestigio, "actos de conocimiento y reconocimiento" (Bourdieu, 2004, p. 26) de los que goza un individuo en la sociedad o en un campo específico. No lo incluimos aquí basándonos en un argumento esgrimido por el propio Bourdieu (1997), cuando señala que se trata simplemente del modo en que los otros tipos de capital (económico, cultural y social) son percibidos socialmente.

${ }^{7} \mathrm{Al}$ afirmar esto Bourdieu rompe con ciertas corrientes sociológicas -en particular el marxismo- que consideran las desigualdades culturales como secundarias respecto de las desigualdades económicas. Estas corrientes reducirían el papel de las -producciones simbólicas a instrumentos de manipulación y dominación política- (Nogueira \& Nogueira, 2009, p. 35). Al entender que los campos de producción simbólica son relativamente autónomos de la economía Bourdieu reconoce plenamente la importancia de las disputas que se dan en ese terreno. 
cepto de capital: los sujetos pueden ser considerados como inversores (aún si no tienen plena conciencia de ello) que operan en diferentes mercados (el mercado de trabajo, el mercado escolar, el mercado matrimonial) convirtiendo valores de un tipo de capital en otro e intentando extraer beneficios de ello. Piénsese, por ejemplo, que un capital social alto puede ayudar a la acumulación de capital económico cuando se pretende iniciar un nuevo emprendimiento, o que, cuando matriculan a sus hijos a una buena escuela privada ${ }^{8}$, las familias de clase media invierten en la acumulación de capital cultural de sus hijos. Considérese también que el capital cultural puede ser invertido en el mercado de trabajo para permitir el acceso a empleos más calificados, lo cual repercute en la acumulación de capital económico. En síntesis, un grado elevado de una forma de capital no garantiza la acumulación de las formas restantes pero la facilita.

\section{Introduciendo el concepto de habi- tus}

Un problema fundamental en sociología es el de la relación entre las acciones de los sujetos y las estructuras socia$\operatorname{les}^{9}$. A ese respecto, podemos identificar dos posiciones extremas igualmente criticadas por Bourdieu: (1) el subjetivis- mo, en el cual las acciones de los sujetos resultan de su libre albedrío y son productoras de las estructuras sociales; y (2) el objetivismo, en que las estructuras sociales se imponen a los individuos y los obligan a actuar en conformidad con ellas. Con el propósito de abordar la cuestión evitando ambos extremos Bourdieu elabora el concepto de habitus.

Para Bourdieu (1983), los individuos tenderían a actuar de acuerdo con el conjunto de disposiciones prácticas (es decir, de inclinaciones a un determinado tipo de comportamiento) típico de los grupos sociales en los que fueron socializados. Este sistema de disposiciones prácticas es llamado habitus, y constituye un concepto tan importante en la teoría de Bourdieu que merece una discusión más detallada.

En primer lugar, debe quedar claro que, por tratarse de disposiciones, el habitus no debe ser entendido como un conjunto de normas inflexibles sino como principios generales que orientan la acción. Así, la estructura social guiaría las acciones individuales sin determinarlas de manera mecánica e inmediata. Entre otras cosas, porque esos principios siempre deberán ser adaptados a las nuevas condiciones objetivas con las que se encuentra el sujeto. La comprensión de este punto es esencial para entender por qué la propuesta de

\footnotetext{
${ }^{8}$ En el sistema educativo brasileño, los egresados de las escuelas privadas de Educación Básica por lo general tienen mayores probabilidades de realizar trayectorias profesionales más prestigiosas.

${ }^{9}$ Son ejemplos importantes de estructuras sociales abordadas por Bourdieu: (1) la estructura general de las relaciones de clase, determinada por la distribución desigual de las tres formas fundamentales de capital (económico, cultural y social); (2) la estructura de las relaciones de fuerza interior a cada campo, determinada por la distribución desigual de una forma específica de capital (científico, burocrático, religioso, etc.) entre los agentes del campo.
} 
Bourdieu constituye una alternativa al objetivismo y al subjetivismo (Nogueira \& Nogueira, 2009).

En segundo lugar, observamos que el mecanismo de formación del habitus de un individuo es la socialización. El conjunto de las vivencias del sujeto en sociedad conduce a la construcción de percepciones y prejuicios que estructuran su subjetividad y orientan así sus acciones. Ese conjunto de vivencias es función de la posición del sujeto en las estructuras sociales. Por lo tanto, el habitus es, en cierta medida, la internalización de las estructuras sociales por parte del sujeto.

Por último, como el habitus tiene su origen en las experiencias anteriores de éxito y fracaso de los diversos grupos sociales (por ejemplo, las experiencias con mayor o menor éxito de cada grupo familiar en la inversión en educación con miras al ascenso social), se observa que el habitus ofrece generalmente al sujeto una orientación adecuada a sus condiciones objetivas, es decir, un conjunto de disposiciones con una considerable adaptación a sus posibilidades reales de éxito (1983).

\section{Histéresis del habitus y trabajo pedagógico}

El habitus se desarrolla a lo largo de todo el proceso de socialización. Esto sig- nifica que nunca es definitivo. Con todo, Bourdieu (1983) observa que los primeros procesos de socialización tienen particular importancia en la conformación del habitus, ya que la forma en que serán percibidas e interpretadas las experiencias sociales posteriores está condicionada por el habitus anterior. Por ello, los cambios en la posición social del individuo no implican cambios inmediatos en sus disposiciones prácticas. Esa tendencia de los sujetos a conservar sus disposiciones prácticas aun cuando su contexto se ha modificado Bourdieu la llama histéresis del habitus ${ }^{10}$. En síntesis, el habitus individual no se corresponde directamente con la posición social actual del sujeto sino que refleja fundamentalmente su origen social.

Una consecuencia de la histéresis del habitus es que la eficacia del trabajo pedagógico ${ }^{11}$ depende siempre de la distancia entre el habitus correspondiente al origen social de los alumnos y el habitus que se desea enseñar (Bourdieu $\&$ Passeron, 2009). Esto tiene diversas implicancias para la educación, y es relevante por ejemplo cuando la escuela intenta promover disposiciones orientadas a la permanencia en los estudios en alumnos de clase popular cuyo habitus familiar privilegia el ingreso precoz en el mercado de trabajo. O cuando se desea inculcar la forma de pensar y resolver problemas propia de las disci-

\footnotetext{
${ }^{10}$ Se puede ilustrar la histéresis del habitus por varias situaciones. Por ejemplo, puede observársela en familias de la burguesía decadente que tienden a conservar su diletantismo aun cuando su capital económico se encuentra severamente restringido. $\mathrm{O}$ en el caso de los alumnos que, en su ingreso a la universidad, necesitan un periodo de adaptación al nivel de dedicación exigido.

${ }^{11}$ Bourdieu y Passeron (2009) definen el trabajo pedagógico como un trabajo extensivo que tiene por objetivo inculcar un conjunto duradero de disposiciones prácticas, es decir, un habitus que permanezca aún después de concluido el trabajo pedagógico.
} 
plinas científicas en alumnos que no han adquirido las habilidades previas que el trabajo pedagógico presupone.

De manera general, dado que los alumnos llegan a la escuela con disposiciones prácticas diversas (en virtud de la heterogeneidad de sus orígenes sociales), un mismo trabajo pedagógico tiende a presentar resultados igualmente variados y con perjuicio para las clases dominadas, en la medida en que (Bourdieu \& Passeron, 2009): (1) sigue patrones rigurosos de homogeneidad y -neutralidad-, que hacen que todos los estudiantes reciban el mismo tratamiento; (2) presupone que todos los alumnos poseen ciertas disposiciones prácticas que en los hechos se encuentran más raramente en los hijos de clases populares.

En las carreras de grado de Física de una universidad brasileña se observó que en las disciplinas introductorias los alumnos de clase popular son retenidos por más tiempo y tienden a obtener calificaciones más bajas (Autores, en imprenta). En efecto, los alumnos de clase popular tienden a ingresar a la carrera de Física con carencia de algunos -conocimientos previos-, lo cual puede de hecho perjudicarlos. Con todo, la propia carencia de conocimientos previos tiene un origen social, y existe toda una serie de otras características comportamentales y actitudinales que, aun si no entran en los objetivos comúnmente llamados de-conocimiento-, pueden ser determinantes para la realización de trayectorias exitosas en la carrera de Física (tales como la permanencia en los estudios aun cuando no hay una perspectiva cierta de retorno inmedia- to). No obstante, todos esos aspectos se encuentran incorporados en el concepto de habitus, y así -en comparación con el tradicional abordaje que pone atención fundamentalmente en el conocimiento previo de los estudiantes en las actividades de enseñanza (Ausubel, 2000)la consideración más general de la distancia entre el habitus adquirido y en habitus por enseñar puede aportar nuevas perspectivas en el planeamiento de actividades, evaluaciones y currículos en educación científica.

\section{Habitus de clase e inversión en el mercado escolar}

$\mathrm{Al}$ modo de inversores en el mercado -que evalúan sus recursos disponibles y trazan estrategias que, en vista de sus experiencias previas, aparecen con más probabilidades de rendirles un retorno mayor- las familias tienden a usar estrategias distintas para invertir en el -mercado escolar-. Aunque exista una gran diversidad, es posible identificar cierta regularidad en las estrategias adoptadas por individuos de una misma clase social, lo cual las caracteriza como parte de su habitus de clase. El análisis de esas estrategias ayuda a comprender de qué modos el origen social de los estudiantes puede producir resultados educativos sensiblemente diferentes.

Habitus de clase popular. Según Bourdieu (2008a), en vista de las restricciones impuestas por la falta de capital de todos los tipos, y dado que las experiencias de éxito escolar en los medios frecuentados por los individuos de esa 
clase son menos frecuentes, las familias de clase popular tienden a invertir en trayectorias escolares cortas y en profesiones que exigen poca escolarización. Para esas familias, la realización de trayectorias escolares prolongadas (como ingresar y concluir una carrera de prestigio) puede significar una inversión sumamente grande e incierta de recursos y esfuerzos. Según Bourdieu, la lógica de las clases populares es la lógica de lo necesario, y así, en su mayoría, los padres de clases populares tenderían a exigir a sus hijos que estudien sólo lo necesario para mantenerse (lo que, habida cuenta de la expansión del acceso al sistema educativo, significa por lo general estudiar más de lo que lo hicieron los padres), o para experimentar un pequeño ascenso social.

Habitus de clase media. En la medida en que nos alejamos de las clases populares y vamos hacia las clases media y dominante, se puede ver que, dentro de cada una de ellas, los diversos tipos de capital se distribuyen de manera diferenciada y que, por lo tanto, es necesario considerar escisiones internas a las clases $^{12}$. Según Bourdieu (2008a), una de las características de las familias de clase media es que viven entre el miedo a volverse de clase popular y el deseo de convertirse en parte de la élite (económica o intelectual). Así, las familias de clase media tienden a trazar estrategias más agresivas de ascenso social y acu- mulación de capital. Entre esas familias, las que tienen un origen de clase popular y lo deben todo al éxito escolar de sus padres y abuelos son más propensas a invertir agresivamente en el mercado escolar, considerándolo como la única vía de ascenso social o de conservación de sus condiciones de existencia.

Bourdieu identifica en las familias de clase media tres disposiciones prácticas características relacionadas mediata o inmediatamente con el éxito escolar diferencial de los miembros de la clase. Esas estrategias son (2008a): (1) el rigorismo ascético, que implica renunciar a placeres y comodidades inmediatos en favor del proyecto de ascenso o conservación de la posición socioeconómica (esta disposición se manifiesta, por ejemplo, entre estudiantes de grado y posgrado que realizan esfuerzos económicos manteniéndose sólo con lo percibido a título de becas o prácticas profesionales, no por falta de oportunidad de mejores salarios sino por elección, como una estrategia que dedica más tiempo a la carrera para aumentar las probabilidades de éxito, para posteriormente transformar esos esfuerzos en confort y privilegio profesional); (2) el control de la fecundidad o malthusianismo (las familias de clase media tienden, más que las familias de las clases populares y dominantes, a controlar el tamaño de la prole como estrategia para concentrar capital y otros esfuerzos en una cantidad reduci-

\footnotetext{
${ }^{12}$ Se considera de clase media tanto al propietario de una pequeña empresa -cuyo poder, en tanto que capitalista stricto sensu, se basa en el capital económico acumulado- como a los miembros más educados de la clase trabajadora (tales como profesores, abogados, ingenieros, administradores ), cuya posición social está garantizada por títulos escolares y por las habilidades específicas que esos títulos indican.
} 
da de hijos y aumentar así sus probabilidades de éxito escolar y profesional); y (3) la buena voluntad cultural, la docilidad y el esfuerzo con que los miembros de esta clase intentan valorizar, consumir y apropiarse de la cultura dominante (este principio puede observarse en la frecuencia de visitas a museos, teatros, o en la particular docilidad con que algunos estudiantes de diferentes edades se someten a las sanciones escolares y procuran deliberada y constantemente la aprobación de sus maestros en materia de comportamiento y conocimiento).

Habitus de clase dominante. Las élites se distinguen de las clases medias por el hecho de que la gran cantidad de capital acumulado (ya sea económico o cultural) los coloca en una posición confortable, y la posibilidad de caer a la condición de clase popular es muy remota. Según Bourdieu (2008a), la lógica general de las prácticas de clase dominante es la del diletantismo y la distinción. De este modo, consciente o inconscientemente, los miembros de las élites (económica o cultural) intentan distinguirse siempre de lo que consideran vulgar en materia de arte, vestuario, deportes, usos del lenguaje, ejercicio profesional. A diferencia de lo que ocurre con la fracción más educada de la clase media, para los hijos de la clase dominante (económica o cultural) la reproducción de la condición de clase de los padres no exige direccionar tantos esfuerzos al mercado escolar, ya sea porque su condición está ligada a la transferencia de capital económico, ya sea porque la gran acumulación de capital cultural de la familia hace del éxito escolar de los hijos algo mucho más natural. Así, se tiende a considerar la escolaridad como un recurso accesorio en la legitimación de una posición social (cultural o económica) que ya está garantizada por herencia.

\section{Consecuencias de la expansión del acceso al sistema educativo}

Otro contexto en que la idea de mercado escolar se muestra valiosa es el análisis de la reciente masificación de la educación formal y sus consecuencias. La mayoría de los países occidentales vive o vivió, a partir de mediados del siglo XX, la esperanza de que la expansión del sistema educativo resultaría en una mejora global de las condiciones de vida de sus ciudadanos (especialmente los de clase popular). No obstante, según Bourdieu (2008b) existen dos procesos relacionados a la expansión del mercado escolar que contribuyen conjuntamente a que el efecto social pretendido de esa expansión de hecho no tenga lugar. Dichos procesos son. (1) la inflación de títulos; (2) la traslación de las distancias.

La inflación de títulos corresponde a la desvalorización de los certificados escolares en virtud del aumento de la cantidad de títulos disponibles en el mercado, sin que tenga lugar un aumento proporcional de las posiciones en el mercado de trabajo que dependen de dichos certificados. En efecto, el valor de los títulos de educación básica y superior se transforma por el propio hecho de que hay más ciudadanos en la escuela (sobre todo de clase media y popular). Así la expansión del sistema educativo no implica necesariamente 
una mejora en las condiciones de vida de las clases populares. Por el contrario, el aumento de las exigencias del mercado de trabajo ha acompañado el aumento de la cantidad de sujetos -educados-disponibles para contratar. Con ello, la mayoría de los ciudadanos (de todas las clases) se vieron obligados a estudiar más para llegar a ocupar las mismas posiciones que sus padres y abuelos lograron conquistar con menos dedicación a la escuela.

En principio, la inflación de los títulos escolares (y la correlativa elevación de la exigencia de certificados escolares para la gran mayoría de las posiciones en el mercado de trabajo) alcanza a todas las clases que dependen de esos títulos para obtener un estatus social. Así, como consecuencia de la inflación de los títulos y del esfuerzo (consciente o inconsciente) de los individuos por mantener el estatus social y el patrón de confort experimentado en la familia, los ciudadanos de las clases media y dominante pasan también a presentar una mayor dedicación escolar (en comparación con la de sus padres) y así buscan títulos más altos (como los de grado y posgrado), acumulan prácticas profesionales, carreras, especializaciones y todo tipo de experiencia escolar y profesional que les permita mantener la competitividad en un mercado inflacionario. El resultado de esa intensificación generalizada de la escolaridad es lo que Bourdieu llama traslación de las distancias (Bourdieu, 2008b): todos los ciudadanos obtienen progresos en su escolarización en relación con la de sus padres y abuelos, no obstante las diferencias de escolaridad entre los individuos de las diferentes clases permanecen, y así la estructura de las relaciones de clase se mantiene aproximadamente igual.

\section{Una agenda de investigación}

Si bien un análisis extensivo de la obra de Bourdieu escapa al objetivo del presente artículo, hemos podido destacar tanto el carácter integrador del marco teórico en cuestión como algunas consecuencias importantes para la investigación en educación científica. En Bourdieu encontramos elementos para: (1) una teoría del conocimiento en general y del conocimiento científico en particular, basada en el argumento de que toda forma de conocimiento considerada legítima es una construcción cultural arbitraria que depende de determinadas relaciones de fuerza y de la disimulación de esas relaciones para el mantenimiento de su legitimidad; (2) una teoría de la génesis y el funcionamiento del campo científico, que nos permite reconocer la ciencia como un mercado dotado de una dinámica relativamente autónoma, y al científico como un inversor en ese mercado; (3) una teoría de la organización de la sociedad en clases, que se distingue de la tradición economicista en tanto reconoce tres formas diferentes de capital; (4) una teoría de la acción, que vincula las prácticas que los sujetos mantienen habitualmente dentro y fuera del contexto escolar con el origen social de esos sujetos; (5) una teoría del aprendizaje, en la medida en que llama la atención sobre el hecho de que la efectividad del trabajo pedagógico depende de la distancia entre el habitus adquirido y el 
habitus por enseñar; (6) una teoría de la educación como inversión, que sitúa las disposiciones de los alumnos al estudio no tanto en su vocación o curiosidad sino en el contexto de sus ambiciones relativamente inconscientes de ascenso social por medio de la escuela; (7) una teoría acerca de la expansión del sistema educativo y sus consecuencias; y (8) una teoría del currículo, en la medida en que permite reconocer que todo currículo es una selección arbitraria de arbitrarios culturales, capaz de dificultar el éxito de los estudiantes con origen de clase popular.

El carácter integrador de la teoría de Bourdieu, que le permite teorizar sobre temas tan diversos como los aquí señalados, posee un gran potencial para la movilización de una cantidad cada vez mayor de investigadores en educación científica. Considerando que las preguntas tienden a ser más duraderas que las respuestas (en el sentido en que la producción de conocimiento involucra la búsqueda de respuestas cada vez más elaboradas para un conjunto aproximadamente igual de cuestiones fundamentales), enumeramos aquí algunas de las muchas posibles consecuencias de la sociología de Bourdieu para el ámbito de la investigación en educación científica, por medio de las siguientes preguntas:

(1) ¿Qué hay de arbitrario en la ciencia? ¿Qué características de las teorías científicas más consagradas reflejan elecciones arbitrarias (conscientes o inconscientes) por parte de los crea- dores y perpetuadores de esas teorías? ¿Cuáles son las estrategias (experimentales, argumentativas, coercitivas) que utilizan los profesores de ciencia y los científicos para imponer la legitimidad de sus ideas disimulando lo arbitrario de la ciencia?

(2) ¿De qué modo las relaciones de fuerza que se establecen dentro de la comunidad científica condicionan el desarrollo de la ciencia? ¿De qué modo la distribución desigual del capital científico y de otras formas de capital pueden contribuir a la emergencia de nuevas ideas o a la conservación de ideas que -habida cuenta de los contundentes argumentos y la evidencia empírica en contra presentados- ya podrían haber sido superadas?

(3) ¿Qué disposiciones prácticas relevantes para el aprendizaje de ciencias traen los estudiantes al aula? ¿Qué relación hay entre esas disposiciones prácticas y el origen social de los estudiantes? ¿De qué modo los profesores pueden tomar en consideración el habitus adquirido por el estudiante, de modo de hacer el trabajo pedagógico más eficiente para todos los alumnos? ¿De qué modo un currículo de tipo tradicional en la enseñanza de ciencias puede contribuir a la eliminación de los alumnos de origen popular?

El marco de Bourdieu -mucho más que las tradicionales teorías cognitivistas sobre la adquisición del conocimiento ${ }^{13}$ - permite expandir los límites de la investigación en educación científi-

${ }^{13}$ Como ejemplo de teoría sobre la -adquisición de conocimiento- podríamos referirnos a la teoría del aprendizaje significativo de Ausubel (2000), de notable importancia histórica, inclusive para algunos investigadores en Enseñanza de Física tanto en Brasil como en otros países de América Latina. 
ca más allá de los muros del aula y de la escuela, mostrando el modo en que las cuestiones sociales más amplias se encuentran imbricadas en el trabajo pedagógico. La sociología de Bourdieu permite formular nuevas preguntas y definir nuevos objetos de investigación que, bajo la perspectiva de una investigación en educación científica restringida al aula, serían impensables.

\section{Bibliografia}

ASSIS, A. (1995). Eletrodinâmica de

Weber: Teoria, aplicações e exercícios.

Campinas: Unicamp.

ASSIS, A. (1998). Mecânica relacional.

Campinas: Unicamp.

AUSUBEL, D. (2000). The acquisition and retention of knowledge. Dordrecht: Kluwer Academic Publishers.

AUTORES. (en imprenta). Revista Bra-

sileira de Pesquisa em Educação em Ciências.

BOURDIEU, P. (1983). Esboço de uma teoria da prática. In: R. Ortiz (Ed.), Pierre Bourdieu: Sociologia. (pp. 46-81). São Paulo: Ática.

BOURDIEU, P. (1996a). Espíritos de

Estado: Gênese e estrutura do campo burocrático. In: P. Bourdieu, Razões práticas: Sobre a teoria da ação (10 ed., pp. 91-123). Campinas: Papirus. BOURDIEU, P. (1996b). Espaço social e espaço simbólico. In: P. Bourdieu, Razões práticas: Sobre a teoria da ação (10 ed., pp. 13-33). Campinas: Papirus.
BOURDIEU, P. (1997). The forms of capital. In: A. e. Halsey (Ed.), Education, culture, economy and society (pp. 46-58). New York: Oxford University Press.

BOURDIEU, P. (2003). Algumas propriedades dos campos. In: P. Bourdieu, Questões de sociologia (pp. 119126). Lisboa: Fim de Século.

BOURDIEU, P. (2004). Os usos sociais da ciência: Por uma sociologia clínica do campo científico. São Paulo: UNESP.

BOURDIEU, P. (2008). Os três estados do capital cultural. In: M. Nogueira, \& A. Catani (Eds.), Escritos de Educação (10 ed., pp. 71-80). Petrópolis: Vozes.

BOURDIEU, P. (2008a). Futuro de classe e causalidade do provável. In: $\mathrm{M}$. Nogueira, \& A. Catani (Eds.), Escritos de educação (pp. 81-126). Petrópolis: Vozes.

BOURDIEU, P. (2008b). Classificação, desclassificação e reclassificação. In: M. Nogueira, \& A. Catani (Eds.), Escritos de educação (10 ed., pp. 145183). Petrópolis: Vozes.

BOURDIEU, P. (2009a). O mercado de bens simbólicos. In: P. Bourdieu, $A$ economia das trocas simbólicas $(6$ ed., pp. 99-192). São Paulo: Perspectiva.

BOURDIEU, P. (2009b). Gênese e estrutura do campo religioso. In: P. Bourdieu, A economia das trocas simbólicas (6 ed., pp. 27-78). São Paulo: Perspectiva.

BOURDIEU, P., \& PASSERON, J. (2009). A reprodução: Elementos 
para uma teoria do sistema de ensino (2 ed.). Petrópolis: Vozes.

CARTER, L. (2008). Sociocultural influences of science education: Inovation for contemporary times. Science Education, 92, pp. 165-181. http://dx.doi.org/10.1002/sce.20228

HOBSBAWN, E. J. (2009). A era das revoluções: 1789 - 1848. São Paulo: Paz e Terra.

LEMKE, J. (2001). Articulating communities: Sociocultural perspectives on
scienceeducation.JournalofResearch in Science Teaching, 38, pp. 296316. http://dx.doi.org/10.1002/1098$2736(200103) 38: 3<296::$ A ID TEA1007>3.0.CO;2-R

NOGUEIRA, M., \& NOGUEIRA, C. (2009). Bourdieu e a educação. Belo Horizonte: Autêntica.

OECD. (2007). PISA 2006: Science competencies for tomorrow's world (executive summary). Paris: OECD. 